DOI 10.18699/SBB-2020-17

\title{
De novo identification and sequence assembly of high-copy tandem repeats in raw data Oxford Nanopore plant DNA sequencing data
}

Kolganova E. ${ }^{1}$, Muravenko O. ${ }^{2}$, Kirov I. ${ }^{1}$

${ }^{1}$ All-Russia Research Institute of Agricultural Biotechnology, Moscow, Russia

${ }^{2}$ Engelhardt Institute of Molecular Biology, Russian Academy of Sciences, Moscow, Russia

*e-mail: liza.colg@gmail.com

Key words: long-reads, ONP, tandem repeats, consensus

Motivation and Aim: High-copy tandemly organized repetitive sequences (TRs) may constitute a large part (up to $30 \%$ ) of plant genomes. They play important role in genome structure organization, cell division, and diversity. Yet, TR functions, origin, evolution, and genome organization are still poorly understood. Assembly of full-size clusters of tandem repeats from short reads can result in reduction in cluster of TRs and large-scale misassembles of sequences and remains a great challenge. Oxford Nanopore Technologies (ONT) directly sequences single molecules of DNA and is capable to generate long reads covering a significant part of individual TR genomic clusters. The exponentially growing of publicly available ONT datasets for plants having no reference genome assembly triggers us to design a pipeline for high-copy TRs identification in raw ONT reads. The relatively high error rate in data of nanopore sequencing compared to short sequencing makes it impossible to directly search for TRs by known tools (e.g. Tandem Repeat Finder) [1]. Therefore, in this work we have developed a pipeline for de novo identification and sequence assembly of high-copy tandem repeats in raw data ONT plant DNA data, to calculate TRs copy number variation and assembly consensus sequences for further analyses (e.g. FISH).

Methods and Algorithms: Pipeline works with Oxford Nanopore Technologies (ONT) sequencing data in FASTA or FASTQ formats. The success of this pipeline work was proven by the analysis of originally obtained Deschampsia antarctica ONT data. We identified more than 40 TRs. Of them, 27 high-copy TRs have been previously identified by several previous studies [2]. The data was used to evaluate the robustness of the pipeline in terms of TR sequence and TR genome abundancy. The results showed a high $(r=0.98$, P-value $<1$ e-15) correlation of TRs copy number estimated by our pipeline and by previous studies. Moreover, 13 new high-copy TRs have been found.

Conclusion: Our pipeline identifies high-copy TRs in raw long-reads. Furthermore, this pipeline will provide insights about novel TRs and increase knowledge about their structural organization and evolution. Source code and instructions for local installation are available at https:/github.com/Kirovez/nanoTRF. Acknowledgements: This work was financially supported by the RFBR (17-00-00336).

\section{References}

1. Benson G. Tandem repeats finder: a program to analyze DNA sequences. Nucleic Acids Research. 1999. Vol. 27 . Issue 2. P. 573-580.

2. González M.L., Chiapella J.O., Urdampilleta J.D. Characterization of some satellite DNA families in Deschampsia antarctica (Poaceae). Polar Biol. 2018. Vol. 41. Issue 3. P. 457-468. 